Supplement of Hydrol. Earth Syst. Sci., 20, 669-683, 2016

http://www.hydrol-earth-syst-sci.net/20/669/2016/

doi:10.5194/hess-20-669-2016-supplement

(C) Author(s) 2016. CC Attribution 3.0 License.

(c) (1)

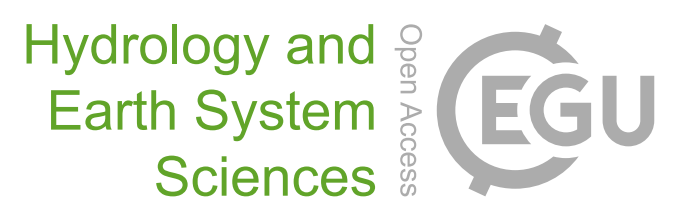

Supplement of

\title{
Comparing statistical and process-based flow duration curve models in ungauged basins and changing rain regimes
}

M. F. Müller and S. E. Thompson

Correspondence to: M. F. Müller (marc.muller@berkeley.edu)

The copyright of individual parts of the supplement might differ from the CC-BY 3.0 licence. 


\title{
Supplementary Materials: \\ Comparing statistical and process-based flow duration curve models in ungauged basins and changing rain regimes
}

\author{
Marc F. Müller, Sally E. Thompson*
}

January 2016

This document contains the supplementary material to the research article Comparing statistical and process-based flow duration curve models in ungauged basins and changing rain regimes by M.F. Müller and S.E. Thompson (2016), published in Hydrology and Earth System Science. Section S1 describes the performance of the Medium Irrigation Project method currently implemented in Nepal to predict Flow Duration Curves (FDC) in ungauged basins for infrastructure design purposes. Section S2 describes supplementary cross-validation analyses conducted to attribute the uncertainty sources of the assessed methods. Section S3 describes the theoretical arguments relating catchment characteristics to the resilience of stream regimes (and therefore the reliability of the statistical method for predictions under change) in seasonally dry climates. Lastly, Section S4 describes the Monte Carlo analysis conducted to test these hypothesized relations.

\section{S1 Performance of the Medium Irrigation Project method in ungauged Nepalese basins}

To benchmark the methods evaluated in this study, we assess the predictive performance of the Medium Irrigation Project, an empirical method currently used to predict streamflow distribution in small mountainous catchments in Nepal for infrastructure design purposes. The method is prescribed by official micro hydropower design guidelines in Nepal Alternative Energy Promotion Center [1]. The approach, described in [3], divides Nepal into seven hydrologic regions characterized by different sets of monthly

\footnotetext{
${ }^{*}$ Department of Civil and Environmental Engineering, Davis Hall, University of California, Berkeley
} CA, USA 
flow indices. Streamflow distribution in ungauged catchments is determined by performing a site visit in mid-April to evaluate discharge under low-flow conditions. In our validation analysis, we emulated this step by selecting the daily flow measured on April $15^{\text {th }}$ of a randomly drawn observation year at each gauge. The measured flow is then used to scale the regional monthly indices corresponding to the location of the catchment. All regions have an index of 1 for the month of April (when low-flow conditions are observed), and larger indices for the other months. FDCs can finally be computed by reordering daily flow values interpolated from the obtained monthly flows.

A fundamental flaw of the method is that it assumes that discharge measured in mid-April on a given year is representative of lowest flow conditions that can be observed in the catchment. This assumption does not hold if effective rain events have occurred shortly before discharge was measured or, more to the point, if the current year is not a particularly dry year. As a result, predicted FDCs substantially overestimate observed flows, as shown in Figure S1 (a), and provide a clear motivation to seek improved, yet tractable prediction options.

\section{S2 Determination of Uncertainty Sources: Supple- mentary Analyses}

\section{S2.1 Statistical Method}

Figure S1 (b) shows that parameter interpolation errors are the main source of uncertainty of the statistical method. Using observed (instead of predicted) flow indices substantially reduces the width of the error-duration curve of the statistical method. The relative sensitivity of the statistical method to interpolation errors in each flow index was evaluated through a numerical simulation (Figure S2 (a)). The prediction performance was more sensitive to errors in $Q_{m}$ than $q_{95}$. This is consistent with the fact that, while $Q_{m}$ has a direct effect on all the quantiles of the FDC, $q_{95}$ only determines the type of FDC shape that is selected, and two different values of $q_{95}$ may generate identical FDC shapes. The statistical approach assumes that catchments with similar low-flow indices $\left(q_{95}\right)$ have identical hydrological responses, and therefore identical FDC shapes. Errors caused by the violation of this assumption also appear in the error duration curve on Figure S1 (b). The statistical method assigns gauges to a finite number of bins, according to their low flow index, and determines a FDC shape for each bin. The error duration curve shown on Figure S1 (b) represents differences in the FDC shapes of catchments within the bins (i.e. with similar low flow indices). A tradeoff arises in determining the number of bins: a small number of large bins leads to large averaging errors within the bins, while a large number of thin bins increases the model's sensitivity to interpolation errors in $q_{95}$. 


\section{S2.2 Process-based Method}

In contrast, interpolation uncertainties on the model parameters ( $k$ and $b$ ) only marginally affect the prediction performance of the process-based approach (Figure S3 (a)). Two other sources of error appear to drive prediction performance. Firstly, uncertainties are caused by the aggregation of point-rainfall statistics to the catchment level. These errors are caused by spatial heterogeneities in wet season rainfall and principally affect high flows, as seen when comparing Figures S3 (b) and (c). Secondly, the numerical analysis presented in Figure $\mathrm{S} 2$ illustrates the crucial importance of dry-season recession constants. While the model is remarkably robust to deviations from key assumptions on rainfall distribution and recession relations [see 4], prediction errors at low flows appear to be caused by errors in the determination of $a$, the scale parameter of the non-linear seasonal recession (Figure S3 (b)). In Müller et al. [4, $a$ is expressed as an explicit function of $k$ and $b$ for sufficiently short recession times, where power-law recessions can be approximated by exponential functions. Although this approach provides more accurate estimates of $a$ than would be obtained through spatial interpolation (Figure S3 (b)), estimation uncertainties remain, propagate through the model and drive prediction errors during the dry season.

The numerical analysis presented in Figure S2 illustrates the crucial importance of dry-season recession constants. Unlike the statistical model, the process-based model is affected by errors in both of its parameters $(k$ and $b)$. However, the model's sensitivity to $k$ is at least partly due to its effect on the estimation of $a$. The sensitivity of $k$ drops if the wet-season recession constant is not used to determine $a$, as seen in Figure S2 (c), where the error introduced on $k$ does not propagate to $a$. This effect is also visible in the resampling analysis on short time series (Figure 3(c) of the main article), where the uncertainty on $k$ only marginally affects prediction performance, which declines when $b$ estimates become inaccurate. This shows that the performance of the model is strongly driven by the estimation of dry-season recession constants in ungauged catchments.

\section{S3 Catchment Characteristics and Streamflow Re- silience in Seasonally Dry Climates}

We examine the linkages between the resilience of flow regimes and the physical characteristics of the catchments. This allows us to identify regions where the statistical method may not provide reliable predictions under change because the flow distribution is is vulnerable to changing rainfall. By explicitly representing runoff generation processes, the stochastic dynamic framework used in the process-based model is an ideal tool to explore the resilience of flow regimes in catchments that follow its basic underlying assumptions on recession behavior. A similar model was used in non-seasonal climates by Botter et al. [2] to relate the resilience of the probability density function of streamflow to observable catchment characteristics. Here we discuss the case 
of seasonally-dry climates, where the characteristics of the seasonal recessions can substantially affect streamflow resilience, here measured as the change in the flow duration curve (in terms of differences in Nash Sutcliffe Coefficient) resulting from a change in rainfall [1]. We use the relations derived in the stochastic dynamic framework [4] to infer the effect of rainfall and recession characteristics on the resilience of flow regimes. This will allow the reliability of the statistical models to be assessed for predictions under change.

During the wet season, flow regimes are determined by the ratio between $\lambda$, the frequency of effective (i.e. runoff-generating) rain events, and $k$ the (linear) recession constant that represents the time scale of the hydrological response of the catchment [2]. If $\lambda / k>1$, frequent effective rainfall and a slow catchment response guarantee a persistent supply of runoff to the stream. If $\lambda / k<1$, effective rain is not frequent enough to compensate for rapid decreases in streamflow after runoff events, and the stream may become intermittent. Streamflow in persistent regimes $(\lambda / k>1)$ is driven by rainfall, whereas streamflow in intermittent regimes $(\lambda / k<1)$ is constrained by the ability of the catchment to modulate the release of water stored in the subsurface. Accordingly, rainfall changes affect most flow quantiles in the persistent regime and shift the entire flow distribution, but they preferentially affect high flows in the intermittent regime, which occur immediately after effective rain events. As a result, intermittent regimes are more resilient to climate change in terms of the mean effect on the entire streamflow distribution, as observed by Botter et al. [2] and illustrated in Figure S4 (a). However, when specifically considering climate effects on the shape of the flow distribution (i.e. by normalizing all flow quantiles by their mean), intermittent regimes are more vulnerable to rainfall changes, which 'tilt' normalized FDCs by preferentially affecting high flows (Figure S4 (b)). Consequently, we expect the statistical method to perform better in persistent flow regimes because the shape of streamflow distribution is less sensitive to changing rainfall. This is confirmed in the Monte Carlo analysis presented in below, where the ratio $\lambda / k$ is positively correlated to the performance of the statistical model.

If no significant rainfall occurs outside of the wet season, climate change only affects dry-season flow through its effect on the initial condition of the seasonal recession. It follows the flow regime will be more sensitive to rainfall changes if the duration of the wet season (when rainfall has a 'direct' effect on streamflow) is long and thus affects a greater proportion of the annual flow duration curve. This effect is also visible in the Monte Carlo analysis, where the duration of the wet season $\left(T_{w}\right)$ is negatively associated with the performance of the statistical model.

The extent to which changes in the initial condition affect the shape of the seasonal recession during the dry season is determined by the non-linear character of the catchment's response. This can be seen by using the characteristic time-scale of the

\footnotetext{
${ }^{1}$ This contrasts with Botter et al. 2], who considers the effect of rainfall regime changes on the probability distribution function of streamflow. While the general idea is the same, the numerical results can be different.
} 
recession (here we consider the time necessary to reduce peak flow by $1 / e$ ) to characterize its shape. In linear catchments, the recession takes an exponential form, so the characteristic timescale corresponds to the inverse of the recession constant and is not affected by initial conditions. For non-linear catchments, characteristic time can be derived from Equation 1 in the main article:

$$
t_{1 / e}=\frac{\left(1-e^{-r}\right) Q_{0}^{r}}{a r}
$$

with $r=1-b$. In these nonlinear regimes, the initial conditions $Q_{0}$ clearly have an effect on the shape of the recession of non-linear catchment. Taking the derivative of Equation

1 with respect to $Q_{0}$ shows that a change in initial flow has a stronger influence on the shape of the recession for low values of $Q_{0}$, as illustrated in Figure S4 (c). Consequently, the sensitivity of the dry season flow regimes to climate change scenarios is expected to be highest in strongly non-linear catchments with limited wet season runoff. Predictions of the FDC using the statistical model for non-stationary rainfall regimes are likely to be poor. In the Monte Carlo analysis below, the performance of the statistical method is significantly worse in strongly non-linear catchments. However, the negative correlation between the linearity of the runoff behavior and the prediction performance is weaker for catchments with high wet-season runoff.

The streamflow resilience in seasonally dry catchments depends on two distinct seasonal effects: a 'direct' effect driven by the ratio between $\lambda_{P}$ and $k$ during the wet season, and an 'indirect' effect during the dry season, when resilience is determined by the interplay between $Q_{0}$ (i.e. wet-season rainfall) and $b$. Streamflow resilience influences the ability of the statistical method to predict FDCs under change. In seasonally dry climates, we expect the statistical method to be most reliable in regions where wet seasons are short with limited total rainfall but persistent flow regimes, and where the recession behavior during the dry-season is close to linear.

\section{S4 Monte Carlo Analysis of Flow Regime Resilience}

We used a Monte Carlo analysis on numerically generated streamflow to estimate the effect of catchment characteristics on streamflow resilience. In the context of this paper, the resilience of flow regimes to climate change is defined as the robustness of the shape of FDCs to shifts in the frequency and intensity of rainfall. The analysis proceeds as follows:

1. Topographic and hydroclimatic characteristics are drawn from uniform distributions as described in Table S1.

2. 1000 years of 'current' synthetic daily streamflows are generated from the drawn parameters using the stochastic rainfall generator and rainfall-runoff model described in Section 2.3.2. of the main article. 
3. Randomly drawn multiplicative biases are inserted to the parameters representing frequency and intensity of rainfall $\left(\lambda_{P}\right.$ and $\left.\alpha_{P}\right)$ to emulate climate change, and 1000 years of 'future' synthetic daily streamflow are generated.

4. Current and future FDCs are constructed empirically from the simulated time series, and normalized by their respective means.

5. Differences in the shape of the flow distributions are quantified by computing the Nash Sutcliffe coefficient on the (log) flow quantiles of the two normalized FDCs.

We repeated the procedure 5000 times and used linear regressions to estimate the effect of catchment characteristics on the resilience of flow regimes, as represented by the Nash Sutcliffe coefficient.

Ordinary least squares estimates of the considered regression models are presented in Table S2. The first column presents direct correlations between catchment characteristics and flow regime resilience and indicate significant positive effects for rainfall frequency and intensity and a negative effect of both recession constants. Regression models shown in columns 2 and 3 test the relations hypothesized in the discussions. As expected, column 2 shows that the $\lambda / k$ ratio has a positive significant effect on the resilience of flow regimes. In order to avoid colinearity issues, all variables used to construct $\lambda$ (i.e. $\lambda_{P}$ and $\alpha_{P}$ in Equation $\mathrm{A} 1$ of the main article) where removed from the regression model. The significant negative effect of $T_{w}$ on streamflow resilience is consistant with the fact that dry season precipitations are neglected. Consequently, changes in rainfall have a direct effect on wet season flows, while only affecting dry season flows through their effect on the initial conditions of the seasonal recession. Lastly, we use mean wet-season rainfall $\left(\lambda_{P} \alpha_{P}\right)$ as a proxy for the initial conditions of the seasonal recession and assess the effect of its interaction with $b$ on flow resilience in column 3 . As expected, $b$ is strongly negatively associated to flow resilience but its interaction with $\lambda_{P} \alpha_{P}$ is significantly positive. This is consistent with our hypothesis that the shape of non-linear recessions is more sensitive to climate, especially if the initial flow conditions are low.

\section{References}

[1] Alternative Energy Promotion Center: Construction and Installation Manual for Micro hydropower Project Installers (In Nepalese), Government of Nepal, 2014.

[2] Botter, G., Basso, S., Rodriguez-Iturbe, I., and Rinaldo, A.: Resilience of river flow regimes, Proceedings of the National Academy of Sciences, 110, doi: 10.1073/pnas.1311920 110, 2013.

[3] Chitrakar, P.: Micro-hydropower design aids manual, Small Hydropower Promotion Project (GTZ) and Mini-grid support program (Alternate Energy Promotion Center, Government of Nepal), 2004. 
[4] Müller, M. F., Dralle, D. N., and Thompson, S. E.: Analytical model for flow duration curves in seasonally dry climates, Water Resources Research, 50, doi:10.1002/2014WR015 301, 2014. 
Table S1: Parameters of the Monte Carlo analysis. At each run, all parameters are drawn independently from a uniform distribution of the specified range.

\begin{tabular}{lll}
\hline \hline Characteristic & Description & Distribution range \\
\hline$k^{-1}[d]$ & $\begin{array}{l}\text { Mean response catchment } \\
\text { response time during the wet season }\end{array}$ & {$[1,10]$} \\
$b[-]$ & $\begin{array}{l}\text { Exponent parameter of the } \\
\text { seasonal recession }\end{array}$ & {$[1,3]$} \\
$T_{w}[d]$ & Wet season duration & {$[50,300]$} \\
$\lambda_{P}^{-1}[d]$ & Mean inter-arrival time of & {$[1,10]$} \\
& wet season rainfall & \\
$\alpha_{P}[m m / d]$ & Mean intensity of wet seasonal rainfall & {$[1,50]$} \\
$\log _{10}(A)\left[\log \left(\mathrm{km}^{2}\right)\right]$ & (Log) catchment area & {$[1,5]$} \\
$r_{\lambda_{P}}[-]$ & Relative change in rainfall frequency & {$[-0.9,0]$} \\
$r_{\alpha_{P}}[-]$ & Relative change in rainfall intensity & {$[0,1]$}
\end{tabular}


Table S2: Linear regression results of the Monte Carlo analysis showing the effect of catchment characteristics on flow resilience. Dependent variables are the Nash Sutcliffe coefficients estimated between the normalized current and future FDCs obtained from the Monte Carlo analysis. Independent variables are constructed from the randomly drawn catchment characteristics listed in Table S1. The first column presents the raw effects of the catchment characteristics on flow resilience. Column 2-3 test the direction and significance of the effects described in Section S3. Superscripted stars describe the significance of the estimated regression coefficients, as described by the $p$-value of Student's $t$ test: ${ }^{* * *}$ indicates a trend different from zero at the $99 \%$ confidence level; ${ }^{* *}$ and ${ }^{*}$ indicate confidence levels of $95 \%$ and $90 \%$ respectively.

\begin{tabular}{|c|c|c|c|}
\hline & \multicolumn{3}{|c|}{ Dependent variable: } \\
\hline & \multicolumn{3}{|c|}{ Nash Sutcliffe Coefficient } \\
\hline & (Baseline) & (Wet. seas) & (Dry seas.) \\
\hline$\lambda_{P}$ & $\begin{array}{c}3.17 \cdot 10^{-1 * * *} \\
\left(2.07 \cdot 10^{-2}\right)\end{array}$ & & \\
\hline$\alpha_{P}$ & $\begin{array}{c}1.49 \cdot 10^{-3 * * *} \\
\left(3.27 \cdot 10^{-4}\right)\end{array}$ & & \\
\hline$k$ & $\begin{array}{c}-4.54 \cdot 10^{-1 * * *} \\
\left(3.32 \cdot 10^{-2}\right)\end{array}$ & & \\
\hline$T_{w}$ & $\begin{array}{c}-3.23 \cdot 10^{-4 * * *} \\
\left(5.72 \cdot 10^{-5}\right)\end{array}$ & $\begin{array}{c}-3.36 \cdot 10^{-4 * * *} \\
\left(5.70 \cdot 10^{-5}\right)\end{array}$ & $\begin{array}{c}-3.68 \cdot 10^{-4 * * *} \\
\left(5.81 \cdot 10^{-5}\right)\end{array}$ \\
\hline$b$ & $\begin{array}{c}-5.75 \cdot 10^{-2 * * *} \\
\left(7.46 \cdot 10^{-3}\right)\end{array}$ & $\begin{array}{c}-6.14 \cdot 10^{-2 * * *} \\
\left(7.42 \cdot 10^{-3}\right)\end{array}$ & $\begin{array}{c}-1.02 \cdot 10^{-1 * * *} \\
\left(1.21 \cdot 10^{-2}\right)\end{array}$ \\
\hline$\lambda / k$ & & $\begin{array}{c}6.28 \cdot 10^{-2 * * *} \\
\left(3.19 \cdot 10^{-3}\right)\end{array}$ & \\
\hline$\lambda_{P} \alpha_{P}$ & & & $\begin{array}{l}-1.31 \cdot 10^{-3} \\
\left(2.19 \cdot 10^{-3}\right)\end{array}$ \\
\hline$\lambda_{P} \alpha_{P}: b$ & & & $\begin{array}{c}4.06 \cdot 10^{-3 * * *} \\
\left(1.03 \cdot 10^{-3}\right)\end{array}$ \\
\hline Observations & 5,000 & 5,000 & 5,000 \\
\hline F Statistic & $559^{* * *}$ & $753^{* * *}$ & $589^{* * *}$ \\
\hline
\end{tabular}


a)

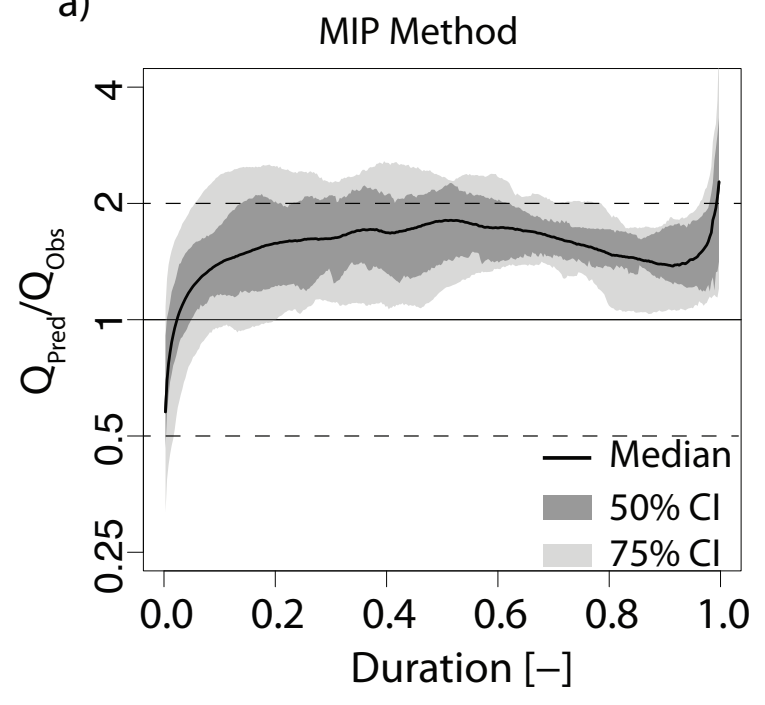

b)

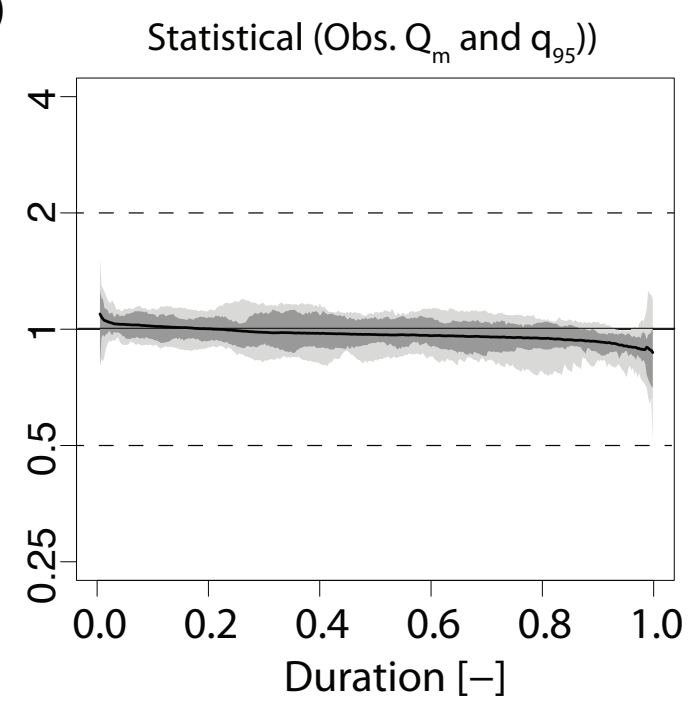

Figure S1: (a) Performance in ungauged basins of the MIP method currently used in Nepal for infrastructure design. The method produces significant upward biases on the predicted FDCs. (b) Error duration curve showing the prediction errors of the statistical methods when the parameters are estimated using observed streamflow, instead of linear regression. Comparison with Figure 2(b) of the main article shows that interpolation uncertainties on the model parameters are the main source of error of the statistical method.
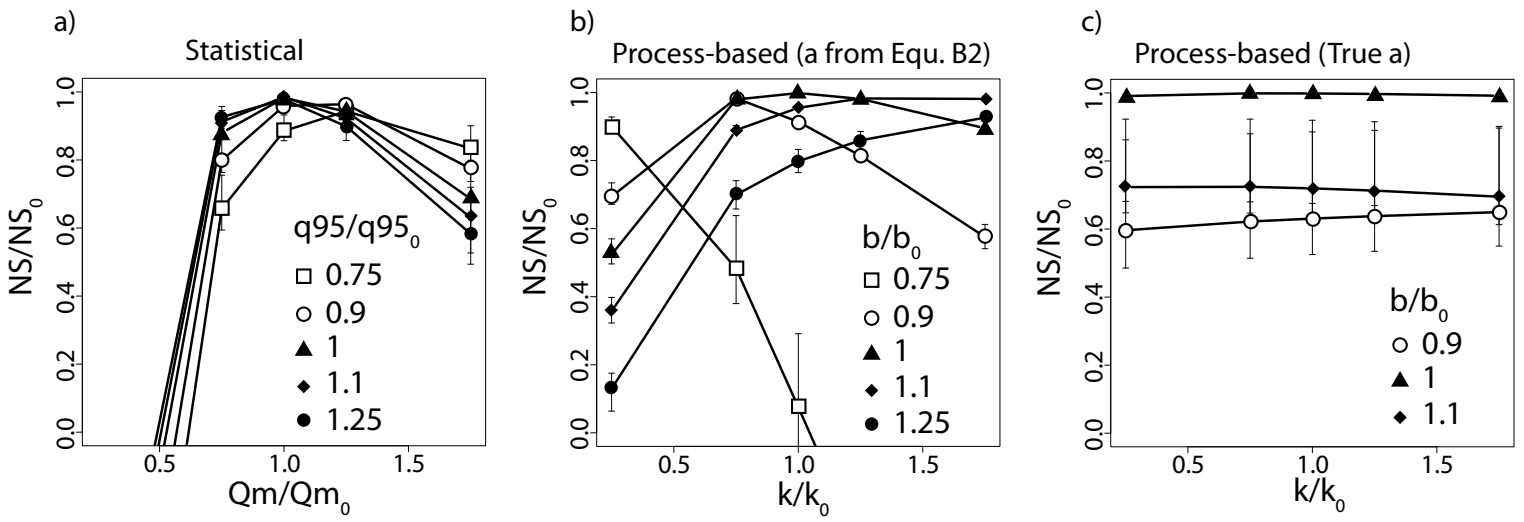

Figure S2: Effect of parameter estimation errors in the predictive performance of the models. Results were obtained using the Monte Carlo analysis described in Section S4. with errors in the parameters inserted instead of rainfall changes. (a) The performance of the statistical model is driven by errors in $Q_{m}$, with little effect of $q_{95}$. (b) The process-based model is sensitive to errors in both of its parameters, but $k$ mostly affects prediction performance through its effect on $a$ (Equation 2 of the main article). (c) Errors in $k$ have little effect on modeling performance if the true values of $a$ are used instead of Equation of the main article. 
a)

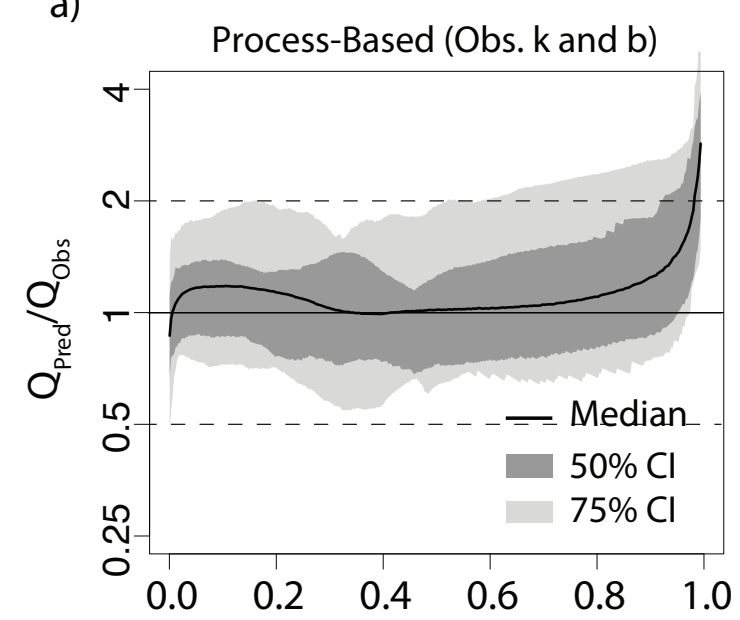

c)

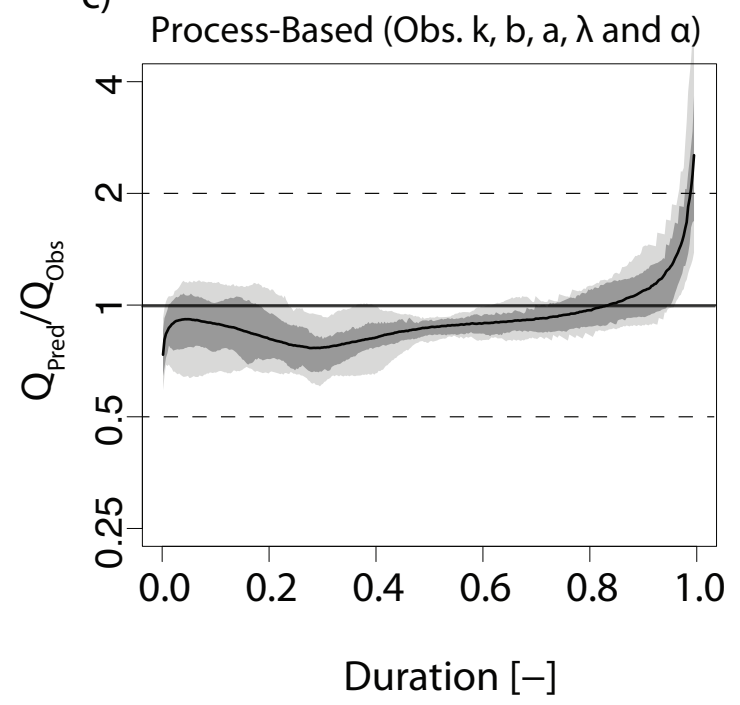

b)

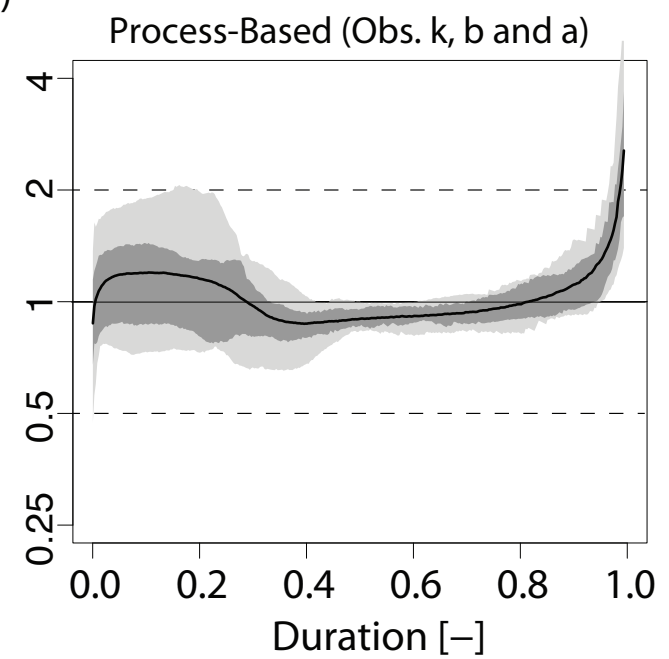

d)

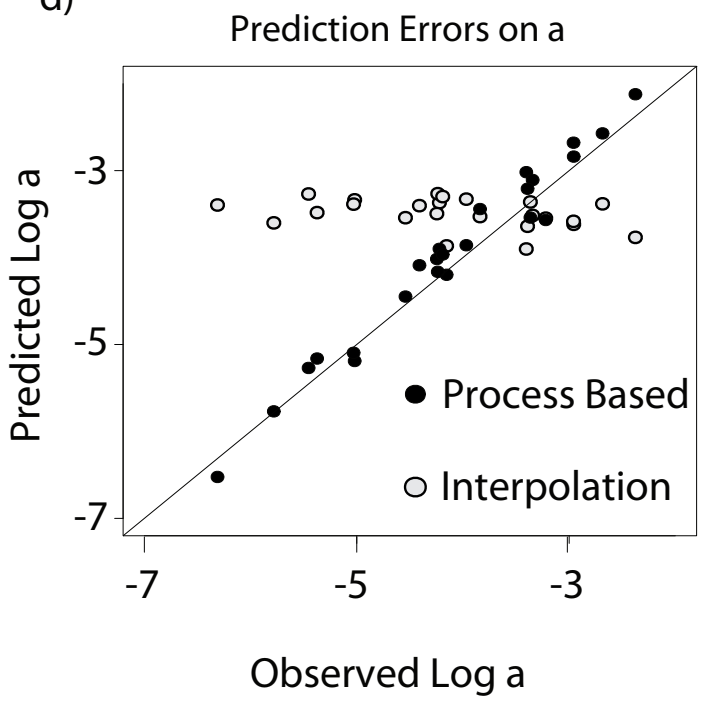

Figure S3: (a - c) Error duration curve of leave-one-out cross-validations for the processbased model using (a) observed values (i.e. estimated from local streamflow observations) for $k$ and $b$, (b) observed values of $k, b$ and $a$, and (c) observed value of $k, b, a, \lambda$ and $\alpha$. Comparing panels (a) to (c) shows that errors in low flow decrease substantially when using observed values for $a$, instead of its approximation from $k$ and $b$ (Equation 2 in main article), whereas errors in high flow decrease substantially when using streamflow, instead of rainfall (see Appendix A of the main article), to estimate $\lambda$ and $\alpha$. (d) Scatterplot of observed vs predicted values for $a$. Predictions errors are small when using Equation 2 of the main article with observed values of $k$ and $b$ (black), and significantly larger when interpolating $a$ from observed values in neighboring catchments using ordinary kriging (grey). 
a)

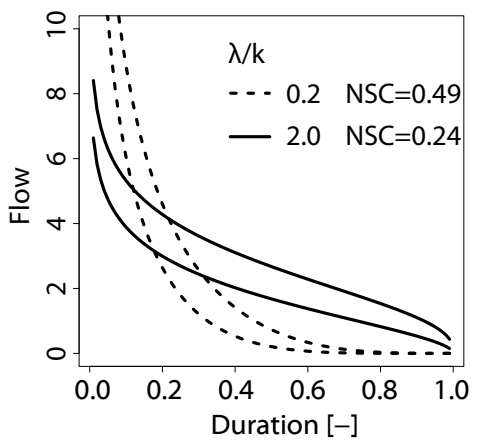

b)

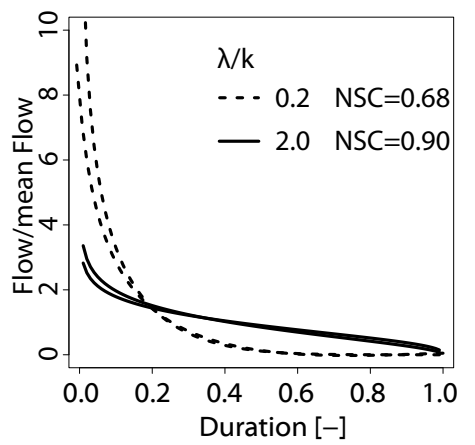

c)

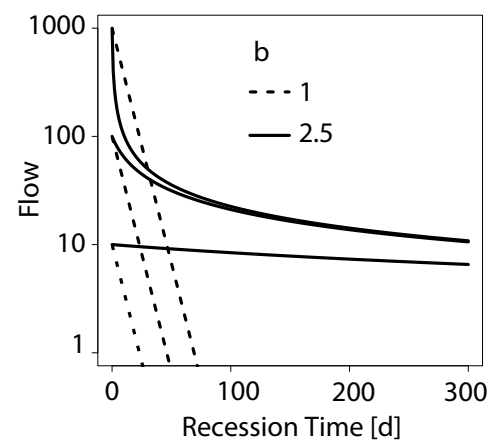

Figure S4: Streamflow resilience to rain changes. (a) Effect of a $50 \%$ increase of rainfall frequency on wet-season FDCs for different flow regimes. The solid FDCs assume a persistant regime $(\lambda / k=2.0)$, with lower and upper curves representing the 'current' and 'future' flow distribution respectively. The dashed curves assume an intermittent regime $(\lambda / k=0.2)$. The persistent regime (solid) is more sensitive to changes than the intermittent regime (dashed), as seen in its lower Nash Sutcliffe Coeffieicnt (NSC) of 0.24. (b) The FDCs presented in panel (a) are normalized by their mean flow. The intermittent regime (dashed) is now more sensitive to change than the resilient regime (solid) because high flows are disproportionately affected by changes in rainfall. (c) Seasonal recession curves for linear (dashed) and non-linear (solid) catchments with different initial flow conditions. The figure illustrate that the shape of the recession is affected by initial conditions only if the catchment recessions are non-linear. For identical relative changes in initial conditions (here 1000\%), the effect on recession shape is most important for low initial flow conditions. 